\title{
Integral Superposition-Type Operators on Some Analytic Function Spaces
}

\author{
A. El-Sayed Ahmed ${ }^{1}{ }^{1}$ and S. Omran ${ }^{2}$ \\ ${ }^{1}$ Mathematics Department, Faculty of Science, Taif University, P.O. Box 11099, Taif 21944, Saudi Arabia \\ ${ }^{2}$ Mathematics Department, Faculty of Science, South Valley University, P.O. Box 83521 Qena, Egypt \\ Correspondence should be addressed to A. El-Sayed Ahmed; ahsayed80@hotmail.com
}

Received 18 January 2021; Revised 7 March 2021; Accepted 19 March 2021; Published 30 March 2021

Academic Editor: Gen Qi Xu

Copyright ( $) 2021$ A. El-Sayed Ahmed and S. Omran. This is an open access article distributed under the Creative Commons Attribution License, which permits unrestricted use, distribution, and reproduction in any medium, provided the original work is properly cited.

All entire functions which transform a class of holomorphic Zygmund-type spaces into weighted analytic Bloch space using the socalled $n$-generalized superposition operator are characterized in this paper. Moreover, certain specific properties such as boundedness and compactness of the newly defined class of generalized integral superposition operators are discussed and established by using the concerned entire functions.

\section{Fundamental Materials}

Let $\mathbb{D}=\{z:|z|<1\}$ be the known concerned open unit disk in $\mathbb{C}$. Assume that $\Xi$ and $\Xi_{1}$ are two concerned metric spaces of holomorphic-type functions both defined on the complex unit disk $\mathbb{D}$. Let $\phi$ be a concerned complex-valued function defined on $\mathbb{D}$. The known superposition operator $S_{\phi}$ on the concerned metric space $\Xi$ is here defined by

$$
S_{\phi}(f)=\phi \circ f, \quad f \in \Xi .
$$

When the operator $S_{\phi} f \in \Xi_{1}$ for $f \in \Xi_{1}$, we call that $\phi$ is induced by the concerned superposition operator from $\Xi$ into $\Xi_{1}$. When the concerned metric space $\Xi$ contains specific concerned linear functions, thus, $\phi$ must be a concerned entire-type function. The symbol $H(\mathbb{D})$ is supposed to be the class of all concerned holomorphic functions on $\mathbb{D}$. For various emerging research studies on the superpositiontype operators, we refer to [1-14] and others.

The motivation of this current study arises from the recent research of the theory of operators on the theory of complex-type function spaces.
The nonlinear $n$-generalized superposition operator can be defined in this current paper by

$$
\left(S_{\phi}^{g, n} f\right)(n, z)=\int_{0}^{z} \phi^{\prime}(f(\xi)) g^{(n-1)}(\xi) d \xi, \quad \text { where } g \in H(\mathbb{D}) \text { and } n \in \mathbb{N}
$$

The operator $S_{\phi}^{g, n}$ will be called the $n$-generalized superposition operator, where

$g^{(n-1)}(z)=\frac{d^{n-1} g(z)}{d z^{n-1}}, \quad$ with" $n-1^{\prime \prime}$-order derivatives, $n \in \mathbb{N}$.

Remark 1. When $n=1$ and $g=f^{\prime}$, the superposition operator is obtained because the difference $S_{\phi}^{g, 1}-S_{\phi}$ is a positive constant. Therefore, $S_{\phi}^{g, n}$ is a generalization of the superpositiontype operator. For good knowledge, the new operator $S_{\phi}^{g, n}$ is defined and discussed in the present concerned manuscript for the first time. 
Remark 2. It should be noted that the graph of the $n$-generalized superposition operator $S_{\phi}^{g, n}$ is almost closed, but because the new operator is a nonlinear operator, then there is no guarantee to establish its boundedness. Nonetheless, there are some spaces $\Xi$ and $\Xi_{1}$, for instance, holomorphictype Bergman, holomorphic Bloch, and holomorphic-type Dirichlet spaces, which implies that the specific function $\phi$ must belong to a specific concerned class of entire-type functions, resulting in the boundedness-type property.

Definition 3 [9]. For a given nondecreasing bounded and continuous function $v:(0,1) \longrightarrow(0, \infty)$, the concerned function $h \in H(\mathbb{D})$ is said to be in the weighted concerned space $\mathscr{B}_{v}$ when

$$
\|h\|_{\mathscr{B}_{v}}:=\sup _{z \in \mathbb{\mathbb { D }}} v(|z|)\left|h^{\prime}(z)\right|<\infty .
$$

Remark 4. In the above definition, suppose that $v(t)=$ $(1-t)^{\alpha}, 0<t<\infty$; then, we obtain the definition of $\alpha$-Bloch space, where $0<\alpha<\infty$.

The following new definition can also be introduced.

Definition 5. Let $h \in H(\mathbb{D})$; then, $h$ is said to be in the weighted little $v$-type concerned Bloch space $B_{v, 0}$ when

$$
\lim _{|z| \longrightarrow 1^{-}} v(|z|)\left|h^{\prime}(z)\right|=0 .
$$

Due to the result of Zygmund in [15] as well as the closed graph theorem, we can deduce that

$$
h \in Z \Longleftrightarrow \sup _{z \in \mathbb{D}}\left(1-|z|^{2}\right)\left|h^{\prime \prime}(z)\right|<\infty .
$$

It is obvious to see that the space $Z$ is a Banach-type space with the specific norm $\|\cdot\|_{Z}$, where

$$
\|h\|_{Z}=|h(0)|+\left|h^{\prime}(0)\right|+\sup _{z \in \mathbb{D}}\left(1-|z|^{2}\right)\left|h^{\prime \prime}(z)\right| .
$$

Here, the concerned Zygmund-type space on $\mathbb{D}$, denoted by $Z_{0}$, is a concerned closed subspace of the holomorphictype space $Z$ that consists of all holomorphic-type functions $h$, for which

$$
\lim _{|z| \longrightarrow 1}\left(1-|z|^{2}\right)\left|h^{\prime \prime}(z)\right|=0
$$

Using (7), it is very obvious to deduce that

$$
\left|h^{\prime}(z)-h^{\prime}(0)\right| \leq C\|h\|_{Z} \log \frac{1}{1-|z|^{2}} .
$$

Some important and essential auxiliary results shall be proved in the next lemmas.
Lemma 6. Let $h \in B_{v}$ and $w \in \mathbb{D}$; then, the following inequality can be obtained:

$$
|h(z)| \leq|h(0)|+\lambda\|h\|_{B_{v}},
$$

for some $\lambda$ which is independent of $h$, with

$$
I_{v}=\int_{0}^{1} \frac{r d r}{v(r)}<\infty .
$$

Proof. Let $|w|>1 / 2, w=r \xi$, and $\xi \in \partial \mathbb{D}$. Then, we infer that

$$
\begin{aligned}
\left|h(w)-h\left(\frac{r \xi}{2}\right)\right| & =\left|\int_{1 / 2}^{1} w h^{\prime}(t w) d t\right| \\
& \leq \int_{1 / 2}^{1}|w|\left|h^{\prime}(t w)\right| d t \\
& \leq 2\|h\|_{B_{v}} \int_{0}^{1} v(|t w|)|w| d t \\
& \leq \lambda\|h\|_{B_{v}} .
\end{aligned}
$$

Additionally, we deduce

$$
|h(w)| \leq \max _{|w| \leq 1 / 2}|h(w)|+C\|h\|_{B_{v}} .
$$

Let $|w| \leq 1 / 2$; then, applying the known mean value theorem for the function $h(w)-h(0)$ (cf. [16]) and considering Jensen's inequality, we deduce that

$$
\begin{aligned}
\max _{|w| \leq 1 / 2}|h(w)-h(0)| & \leq 4^{n} \int_{|h| \leq 3 / 4}|h(w)-h(0)| d A(w) \\
& \leq 4^{n} \int_{|w| \leq 3 / 4}\left|h^{\prime}\left(w_{1}\right)\right|^{2} d A\left(w_{1}\right) \\
& \leq 3^{n} \max _{|w| \leq 3 / 4}\left|h^{\prime}\left(w_{1}\right)\right|^{2},
\end{aligned}
$$

where the homogeneous representation expansion of the function $h$ results in the second inequality.

Therefore,

$$
\begin{aligned}
\max _{|w| \leq 1 / 2}|h(w)| & \leq|h(0)|+(\sqrt{3})^{n} \max _{|w| \leq 3 / 4}\left|h^{\prime}(w)\right| \\
& \leq|h(0)|+(\sqrt{3})^{n} v\left(\frac{3}{4}\right)\|h\|_{B_{v}} .
\end{aligned}
$$

Combining (13) and (15), we deduce

$$
\begin{aligned}
|h(w)| & \leq|h(0)|+(\sqrt{3})^{n} v\left(\frac{3}{4}\right)\|h\|_{B_{v}}+\lambda\|h\|_{B_{v}} \\
& \leq|f(0)|+\left((\sqrt{3})^{n} v\left(\frac{3}{4}\right)+\lambda\right)\|h\|_{B_{v}} .
\end{aligned}
$$

The proof is therefore established completely. 
Hereafter, the letter $\Gamma$ can be used to denote a concerned planar domain and $\partial \Gamma$ defines its boundary.

In view of the known Riemann mapping theorem [16], for any $\Gamma$, there exists a holomorphic function $h$ (that can be called a concerned Riemann mapping) which maps $\mathbb{D}$ onto $\Gamma$ and the concerned origin to a prescribed concerned specific point. The known Euclidean metric of the specific point $w$ to the concerned boundary $\Gamma$ is denoted by $d(w, \partial$ $\Gamma)$; also, the concerned Riemann-type mapping $h$ has the next interesting concerned property:

$\frac{1}{4} v(|w|)\left|h^{\prime}(w)\right| \leq d(h(w), \partial \Gamma) \leq v(|z|)\left|h^{\prime}(w)\right|, \quad$ for all $w \in \mathbb{D}$

which is comparatively similar to the concerned constructions of the concerned connected domains as the concerned images for specific classes of functions in different analytictype function spaces can be obtained in [4].

\section{Auxiliary Results}

The next lemmas establish some elementary properties of the $v$-Bloch-type spaces of analytic functions.

Lemma 7. Let $h \in H(\mathbb{D})$, and assume that (11) holds. Then, for the $v$-Bloch space $B_{v}$, we have the following:

(i) Every bounded concerned sequence $\left(h_{n}\right) \in B_{v}$ is uniformly bounded on concerned compact sets

(ii) For any concerned sequence $\left(h_{n}\right)$ on $B_{v}$ with $\left\|h_{n}\right\|_{B_{v}}$ $\longrightarrow 0$, then $h_{n}-h_{n}(0) \longrightarrow 0$ and the convergence is of uniform type on concerned compact sets

Proof. If $w \in U(0, r), r \in(0,1)$; thus, the following inequalities can be deduced

$\left|h_{n}(w)-h_{n}(0)\right|=\left|\int_{0}^{1} h_{n}^{\prime}(t w) w d t\right| \leq\left\|h_{n}\right\|_{B_{v}} \int_{0}^{1} \frac{|t w| d t}{v(|t w|)} \leq C\left\|h_{n}\right\|_{B_{v}}$.

Hence, the result follows.

Lemma 8. Assume that $h, g \in H(\mathbb{D})$ and $\phi: \mathbb{D} \longrightarrow \mathbb{D}$. Thus, for the n-generalized superposition operator $S_{\phi}^{g, n}: B_{v} \longrightarrow B_{v}$ which is a concerned compact operator and $\Longleftrightarrow S_{\phi}^{g, n}: B_{v}$ $\longrightarrow B_{v}$ which is actually bounded for any bounded concerned sequence $\left(h_{n}\right)_{n \in N} \in B_{v}$ with $h_{n} \longrightarrow 0$ uniformly bounded on concerned compact sets if $n \longrightarrow \infty$, we obtain $\left\|S_{\phi}^{g, n} h_{n}\right\|_{B_{v}}$ $\longrightarrow 0$ as $n \longrightarrow \infty$.

Proof. Now, the concerned assertions (7), (9), and (11) of Lemma 3.7 in [17] can be satisfied for the analytic-type $B_{v}$ space. In view of Lemma 7, thus, it is clear to show that the concerned assertions (i) and (ii) hold. To clear that (ii) holds, assume that the specific sequence $\left(h_{n}\right)$ can be taken as a concerned sequence in the specific closed unit ball of $B_{v}$. Therefore, using Lemma 7 implies that $\left(h_{n}\right)$ can be seen as uniformly bounded on concerned compact sets. Hence, applying the theorem of Montel [18], we can get a concerned subsequence $\left(h_{n_{k}}\right)\left(n_{1}<n_{2}<\cdots\right)$, for which $h_{n_{k}} \longrightarrow h$ which indeed is uniformly bounded on concerned compact sets, for some concerned functions $h^{*} \in H(\mathbb{D})$. Then, it is enough to clear that $h^{*} \in B_{v}$. From Fatou's theorem [16] and the concerned assumptions, we deduce that

$v(|w|)\left|\left(h^{*}\right)^{\prime}(w)\right|=\lim _{k \longrightarrow \infty} v(|w|)\left|\left(h_{n_{k}}\right)^{\prime}(w)\right| \leq \lim _{k \longrightarrow \infty}\left\|h_{n_{k}}\right\|_{B_{v}}<\infty$.

Therefore, in view of Lemma 3.7 in [17], we infer that the $n$-generalized superposition operator $S_{\phi}^{g, n}: B_{v} \longrightarrow B_{v}$ is compact $\Leftrightarrow$ for any bounded concerned sequence $\left(h_{n}\right) \in B_{v}$ with $h_{n} \longrightarrow 0$ uniformly bounded on compact sets as $n \longrightarrow$ $\infty,\left|h_{n}(\phi(0))\right|+\left\|S_{\phi}^{g, n} h_{n}\right\|_{B_{v}} \longrightarrow 0$, as $n \longrightarrow \infty$. Thus, the proof of Lemma 8 is clearly obtained.

Lemma 9. Let $h \in H(\mathbb{D})$. The concerned closed set $K \in B_{v, 0}$ is compact iff it is bounded and satisfies the next concerned condition:

$$
\lim _{|w| \longrightarrow 1} \sup _{h \in K} v(|w|)\left|h^{\prime}(w)\right|=0
$$

Proof. Assume that $K$ is compact, and let $\varepsilon>0$; thus, the specific balls that centered at the specific elements of the concerned $K$ with radii $\varepsilon / 2$ cover $K$; then, by the help of compactness, we can find functions $h_{1}, h_{2}, \cdots, h_{n} \in K$, for which the analytic function $h \in K$ yields that $\left\|h-h_{j}\right\|_{B_{v}}<$ $\varepsilon / 2$ for some $1 \leq j \leq n$; hence,

$$
v(|w|)\left|h^{\prime}(w)\right| \leq v(|w|)\left|f_{j}^{\prime}(w)\right|+\frac{\varepsilon}{2}
$$

Now, for each $j$, we can find $r_{j} \in(0,1)$, for which

$$
v(|w|)\left|h_{j}^{\prime}(w)\right|<\frac{\varepsilon}{2}, \quad \text { whenever } r_{j}<|w|<1 \text {. }
$$

By letting $r=\max \left\{r_{1}, \cdots, r_{n}\right\}$, we obtain

$v(|z|)\left|h^{\prime}(w)\right| \leq \varepsilon, \quad$ whenever $r<|w|<1$ and $h \in K$.

This establishes that (20) holds.

On the other hand, assume that $K \subset B_{v, 0}$ is closed and bounded and satisfies (20).

Then, the set $K$ can be a normal family. When $\left(h_{n}\right)$ is a concerned sequence in the set $K$, then passing to a specific subsequence, we can suppose that $h_{n} \longrightarrow h$ with the concerned uniformly converging on the concerned compact 
subsets of $\mathbb{D}$. Hence, we can clear that $h_{n} \longrightarrow h$ in $B_{v, 0}$. Then, for a given $\varepsilon>0$, using (20), we can find $r \in(0,1)$, for which

$$
v(|z|)\left|h^{\prime}(w)\right| \leq \frac{\varepsilon}{2}, \quad \text { for all } r<|w|<1 \text { and all } h \in K .
$$

Since the convergence $h_{n}^{\prime} \longrightarrow h^{\prime}$ is uniformly bounded on the concerned compact subsets of the disk $\mathbb{D}$, it yields that the type of convergence for $h_{n}^{\prime} \longrightarrow h^{\prime}$ is pointwise on $\mathbb{D}$. Additionally, we deduce that

$$
v(|w|)\left|h^{\prime}(w)\right| \leq \frac{\varepsilon}{2}, \quad \text { for all } r<|z|<1 .
$$

Therefore,

$$
v(|w|)\left|h_{n}^{\prime}(w)-h^{\prime}(w)\right| \leq \varepsilon, \quad \forall r<|w|<1 .
$$

Because the convergence $h_{n}^{\prime} \longrightarrow h^{\prime}$ is uniformly bounded on $r \overline{\mathbb{D}}$, then we can find a specific positive integer $N$, for which

$$
\left|h_{n}^{\prime}(w)-h^{\prime}(w)\right| \leq \varepsilon, \quad \text { for all }|w| \leq r \text { and } n \geq N .
$$

Hence,

$$
v(|w|)\left|h_{n}^{\prime}(w)-h^{\prime}(w)\right| \leq \varepsilon, \quad \forall w \in \mathbb{D} \text { and all } n \geq N .
$$

Hence, $h_{n} \longrightarrow h$ in $B_{v}$. Because $K$ is specifically closed, then we can infer that $h \in K$. Therefore, we deduce that the concerned set $K$ is a compact set. The proof of Lemma 9 is therefore completely established.

\section{Superpositions on Bloch and Zygmund Spaces}

In this section, some interesting and important properties of the new class of the $n$-generalized superposition $S_{\phi}^{g, n}$ acting from the analytic-type Zygmund classes to the analytic $v$ -Bloch classes are clearly established.

Theorem 10. Let $h, g \in H(\mathbb{D})$ and $\phi: \mathbb{D} \longrightarrow \mathbb{D}$. Then, the $n$ -generalized superposition operator $S_{\phi}^{g, n}: Z \longrightarrow B_{v}$ is bounded $\Longleftrightarrow$

$$
F:=\sup _{w \in \mathbb{D}} v(|w|)\left|g^{(n-1)}(w)\right| \log \frac{1}{1-|h(w)|^{2}}<\infty .
$$

Proof. Assume that (29) holds. Hence, for arbitrary $w \in \mathbb{D}$ and $h \in Z$, we deduce that

$$
\begin{aligned}
v(|w|)\left|\left(S_{\phi}^{g, n} h\right)^{\prime}(w)\right| & =v(|w|)\left|g^{(n-1)}(w)\right|\left|\phi^{\prime}(h(w))\right| \\
& \leq C\|\phi\|_{Z} v(|w|)\left|g^{(n-1)}(w)\right| \log \frac{1}{1-|h(w)|^{2}} .
\end{aligned}
$$

From the above inequality (29) and because $S_{\phi}^{g, n} h(0)=0$, we deduce that the operator

$$
S_{\phi}^{g, n}: Z \longrightarrow B_{v}
$$

is a bounded operator.

On the other hand, suppose that $S_{\phi}^{g, n}: Z \longrightarrow B_{v}$ is bounded. Set

$$
f(w)=(w-1)\left[\left(1+\log \frac{1}{1-w}\right)^{2}+1\right],
$$

and also set the function

$$
h_{b}(w)=\frac{f(\bar{b} w)}{\bar{b}}\left(\log \frac{1}{1-|b|^{2}}\right)^{-1},
$$

where $b \in \mathbb{D}$ with $1 / \sqrt{2}<|b|<1$. Then, the easy calculation gives

$$
\begin{aligned}
h_{b}^{\prime}(w) & =\left(\log \frac{1}{1-\bar{b} w}\right)^{2}\left(\log \frac{1}{1-|b|^{2}}\right)^{-1}, \\
h^{\prime \prime}{ }_{b}(w) & =\frac{2 \bar{b}}{1-\bar{b} w}\left(\log \frac{1}{1-\bar{b} w}\right)^{2}\left(\log \frac{1}{1-|b|^{2}}\right)^{-1},
\end{aligned}
$$

which yields that

$$
h^{\prime \prime}{ }_{b}(w)=\frac{2}{1-|w|}\left(C+\log \frac{1}{1-|b|}\right)^{2}\left(\log \frac{1}{1-|b|^{2}}\right)^{-1} \leq \frac{C}{1-|w|},
$$

with $1 / \sqrt{2}<|b|<1$ and $\sup _{1 / \sqrt{2}<|b|<1}\left\|h_{b}\right\|_{Z}<\infty$. Hence, the following inequality can be deduced:

$$
\begin{aligned}
\left\|S_{\phi}^{g, n} h_{\phi(b)}\right\|_{B_{v}} & =v(|w|)\left(S_{\phi}^{g} h_{\phi(b)}\right)^{\prime}(w) \mid \\
& \geq v(|b|)\left|g^{(n-1)}(b)\right| \log \frac{1}{1-|h(b)|^{2}} .
\end{aligned}
$$

Hence, by using the maximum modulus principle, we obtain (29). The proof is therefore completely established.

For the next theorem, the analytic s-Bloch-type space is considered as follows:

$$
\|h(w)\|_{B^{s}}=\sup _{w \in \mathbb{D}}\left(1-|w|^{2}\right)^{s}\left|h^{\prime}(w)\right|<\infty, \quad \text { where } 0<s<1 .
$$

Theorem 11. Assume that $v(|w|)=\left(1-|w|^{2}\right)^{s}$, where $0<$ 
$s<1$. Suppose that $h, g \in H(\mathbb{D})$ and $\phi: \mathbb{D} \longrightarrow \mathbb{D}$. Then, the operator $S_{\phi}^{g, n}: Z \longrightarrow B^{s}$ is a compact operator iff $S_{\phi}^{g, n}: Z$ $\longrightarrow B^{s}$ is bounded, and the following condition holds:

$$
\lim _{|h(w)| \rightarrow 1}\left(1-|w|^{2}\right)^{s}\left|g^{(n-1)}(w)\right| \log \frac{1}{1-|h(w)|^{2}}=0 .
$$

Proof. Assume the boundedness for the operator $S_{\phi}^{g, n}: Z$ $\longrightarrow B^{s}$ holds as well as condition (38) holds too. Therefore, since the operator $S_{\phi}^{g, n}$ is bounded with $\phi(w)=w$, we deduce that

$$
M_{1}:=\left(1-|w|^{2}\right)^{s}\left|g^{(n-1)}(w)\right|<\infty
$$

Let $\left(h_{k}\right)_{k \in \mathbb{N}}$ be a concerned sequence in the Zygmund space $Z$, for which

$$
\sup _{k \in \mathbb{N}}\left\|h_{k}\right\|_{Z} \leq \eta, \quad \text { where } \eta \text { is a positive constant, }
$$

and $h_{k} \longrightarrow 0$ is uniformly bounded on a concerned compact subset of $\mathbb{D}$ as $k \longrightarrow \infty$. Using (38), we obtain that for every $\varepsilon>0$, there is a constant $\delta \in(0,1)$, such that $\delta$ $<|h(w)|<1$, which implies that

$$
\left(1-|w|^{2}\right)^{s}\left|g^{(n-1)}(w)\right| \log \frac{1}{1-|h(z)|^{2}}<\frac{\varepsilon}{\eta} .
$$
that

Now, set $k=\left\{w_{1} \in \mathbb{D}:\left|w_{1}\right|<\delta\right\}$. By (9), we deduce

$$
\begin{aligned}
& \left\|\left.\right|_{\phi} ^{g, n} h_{k}\right\|_{B^{s}}=\sup _{w \in \mathbb{D}}\left(1-|w|^{2}\right)^{s}\left|\phi_{k}^{\prime}(h(w))(g(w))^{(n-1)}\right| \\
& \leq \sup _{h(w) \leq \delta}\left(1-|w|^{2}\right)^{s}\left|\phi_{k}^{\prime}(h(w))\right|\left|g^{(n-1)}(w)\right| \\
& +\sup _{\delta<h(w) \leq 1}\left(1-|w|^{2}\right)^{s}\left|\phi_{k}^{\prime}(h(w))\right|\left|g^{(n-1)}(w)\right| \\
& \leq M_{1} \sup _{w_{1} \in k}\left|\phi_{k}^{\prime}\left(w_{1}\right)\right| \\
& +C\left\|\phi_{k}\right\|_{z<\langle(w) \leq 1} \sup _{\delta<1}\left(1-|w|^{2}\right)^{s}\left|g^{(n-1)}(w)\right| \log \frac{1}{1-|h(w)|^{2}} \\
& \leq M_{1} \sup _{w_{1} \in k}\left|\phi_{k}^{\prime}\left(w_{1}\right)\right|+\varepsilon C \text {. }
\end{aligned}
$$

Applying the known Cauchy's estimate, when we have the specific sequence $\left(h_{k}\right)_{k \in \mathbb{N}}$ that has a uniform type that converges to zero on a specific compact concerned subset of $\mathbb{D}$, we obtain that the specific sequence $\left(\phi_{k}^{\prime}\right)_{k \in \mathbb{N}}$ converges to zero on a specific compact concerned subset of $\mathbb{D}$ as $k \longrightarrow \infty$. Because $k$ is actually compact, it yields that

$$
\lim _{k \rightarrow \infty} \sup _{w_{1} \in k}\left|\phi_{k}^{\prime}\left(w_{1}\right)\right|=0 \text {. }
$$

Letting $k \longrightarrow \infty$ in the last inequality, we deduce that

$$
\lim _{k \longrightarrow \infty} \sup \left\|S_{\phi}^{g, n} h_{k}\right\|_{B^{s}} \leq \varepsilon C .
$$

Because the number $\varepsilon$ is an arbitrary and positive, this yields that

$$
\lim _{k \longrightarrow \infty} \sup \left\|S_{\phi}^{g, n} h_{k}\right\|_{B^{s}}=0
$$

The concerned specific implication is therefore proved.

On the other hand, assume that $S_{\phi}^{g, n}: Z \longrightarrow B^{s}$ is a compact operator. Remarking that the function $h_{b}$ given by (33) has a uniform type that converges to zero on a specific compact concerned subset of $\mathbb{D}$ as $|b| \longrightarrow 1$. In addition, we have

$$
\phi_{b}^{\prime}(b)=\log \frac{1}{1-|b|^{2}}, \quad \text { for each } b \in \mathbb{D} \backslash\{0\} .
$$

Let $\left(w_{k}\right)_{k \in \mathbb{N}}$ be a concerned specific sequence in the disk $\mathbb{D}$, for which $\left|h\left(w_{k}\right)\right| \longrightarrow 1$ when $k \longrightarrow \infty$. The test function $\left(\phi_{k}\right)_{k \in \mathbb{N}}$ can be defined by

$$
\begin{aligned}
\phi_{k}(w)= & \frac{\bar{h}\left(w_{k}\right) w-1}{\bar{h}\left(w_{k}\right)}\left[\left(1+\log \frac{1}{1-\bar{h}\left(w_{k}\right) z}\right)^{2}+1\right] \\
& \cdot\left(\log \frac{1}{1-\left|h\left(w_{k}\right)\right|^{2}}\right)^{-1} .
\end{aligned}
$$

From the proof steps in Theorem 10, we deduce that $\sup _{k \in \mathbb{N}}\left\|\phi_{k}\right\|_{Z} \leq C$. Furthermore, the specific function $\phi_{k}$ converges to zero uniformly on a concerned compact subset of the disk $\mathbb{D}$. Then, using Lemma 7, we obtain that the operator $\left\|S_{\phi}^{g, n} h_{k}\right\|_{B^{s}} \longrightarrow 0$, when $k \longrightarrow \infty$. Moreover,

$$
\begin{aligned}
\left\|S_{\phi}^{g, n} h_{k}\right\|_{B^{s}} & =\sup _{w \in \mathbb{D}}\left(1-\left|w_{k}\right|^{2}\right)^{s}\left|\phi_{k}^{\prime}\left(h\left(w_{k}\right)\right)\left(g\left(w_{k}\right)\right)^{(n-1)}\right| \\
& \geq\left(1-\left|w_{k}\right|^{2}\right)^{s}\left|\phi_{k}^{\prime}\left(h\left(w_{k}\right)\right)\right|\left|g^{(n-1)}\left(w_{k}\right)\right| \\
& \geq\left(1-|w|^{2}\right)^{s}\left|g^{(n-1)}(w)\right| \log \frac{1}{1-|h(w)|^{2}} .
\end{aligned}
$$

Therefore, we can obtain

$$
\lim _{k \longrightarrow \infty}\left(1-\left|w_{k}\right|^{2}\right)^{s}\left|g^{(n-1)}\left(w_{k}\right)\right| \log \frac{1}{1-\left|h\left(w_{k}\right)\right|^{2}}=0 .
$$
clearly.

Thus, the concerned proof of our result is obtained

Now, we give the following characterizations concerning the boundedness of the $n$-generalized superposition operator $S_{\phi}^{g, n}: Z \longrightarrow B_{v, 0}$ on the boundary of the unit disk.

Theorem 12. Assume that $v(|w|)=\left(1-|w|^{2}\right)^{s}$, where $0<s$ $<1$. Suppose that $h, g \in H(\mathbb{D})$ and $\phi: \mathbb{D} \longrightarrow \mathbb{D}$. Then, the 
operator $S_{\phi}^{g, n}: Z \longrightarrow B_{0}^{s}$ is a bounded operator iff condition (38) holds as well as

$$
\lim _{|w| \longrightarrow 1}\left(1-|w|^{2}\right)^{\alpha}\left|g^{(n-1)}(w)\right|=0
$$

Proof. First, assume that conditions (38) and (50) hold. Using condition (38), thus, for every constant $\varepsilon>0$, we can get $r$ $\in(0,1)$, for which

$$
\left(1-|w|^{2}\right)^{s}\left|g^{(n-1)}(w)\right| \log \frac{1}{1-|h(w)|^{2}}<\varepsilon,
$$

with $r<|h(w)|<1$. By (38), we can get $\rho \in(0,1)$, for which

$$
\left(1-|w|^{2}\right)^{s}\left|g^{(n-1)}(w)\right|<\frac{\varepsilon}{\log \left(1 /\left(1-r^{2}\right)\right)},
$$

when $\rho<|w|<1$.

Therefore, when $\rho<|w|<1$ and $r<|h(w)|<1$, we deduce that

$$
\left(1-|w|^{2}\right)^{s}\left|g^{(n-1)}(w)\right| \log \frac{1}{1-|h(w)|^{2}}<\varepsilon
$$

If $\rho<|w|<1$ and $|h(z)| \leq r$, then we can infer that

$$
\begin{aligned}
(1 & \left.-|w|^{2}\right)^{s}\left|g^{(n-1)}(w)\right| \log \frac{1}{1-|h(w)|^{2}} \\
& <\left(1-|w|^{2}\right)^{s}\left|g^{(n-1)}(w)\right| \log \frac{1}{1-r^{2}}<\varepsilon .
\end{aligned}
$$

From (53) and (54), we deduce

$$
\lim _{|w| \longrightarrow 1}\left(1-|w|^{2}\right)^{s}\left|g^{(n-1)}(w)\right| \log \frac{1}{1-|h(w)|^{2}}=0 .
$$

From (55), using the known maximum modulus theorem as well as Theorem 10, the concerned property of boundedness for the concerned operator $S_{\phi}^{g, n}: Z \longrightarrow B^{s}$ can be obtained.

Assuming $f \in Z$ and using (9), we get

$$
\left(1-|w|^{2}\right)^{s}\left|\left(S_{\phi}^{g, n}\right)^{\prime}(w)\right| \leq C\|\phi\|_{Z}\left(1-|w|^{2}\right)^{s}\left|g^{(n-1)}(w)\right| \log \frac{1}{1-|h(w)|^{2}} .
$$

In view of (55), we can deduce that the operator $S_{\phi}^{g, n} \in B_{0}^{s}$, for each $h \in Z$. Because $B_{0}^{s}$ is a concerned closed subset of the analytic space $B^{s}$, it follows therefore that

$$
S_{\phi}^{g, n}(w) \subseteq B_{0}^{s}
$$

Hence, the boundedness of the operator $S_{\phi}^{g, n}: Z \longrightarrow B_{0}^{s}$ can be obtained immediately.

For the converse direction of the proof, assuming that the operator $S_{\phi}^{g, n}: Z \longrightarrow B_{0}^{s}$ holds the boundedness property, then for $\phi(z)=z$, we infer that (50) holds. Now suppose that the concerned condition (38) does not hold. Therefore, we can have $\varepsilon_{0}>0$ and a concerned sequence $\left(w_{k}\right)_{k \in \mathbb{N}} \in \mathbb{D}$, for which $\lim _{k \rightarrow \infty}\left|h\left(w_{k}\right)\right|=1$. Also, we have

$$
\left(1-\left|w_{k}\right|^{2}\right)^{s}\left|g^{n-1}\left(w_{k}\right)\right| \log \frac{1}{1-\left|h\left(w_{k}\right)\right|^{2}} \geq \varepsilon_{0}>0
$$

Then, by choosing a sufficiently large $k$, the following specific inequalities can be directly obtained:

$$
\frac{1-\left|h\left(w_{k-1}\right)\right|}{2}>1-\left|h\left(z_{k}\right)\right|, \quad k \in \mathbb{N} .
$$

Then, for every nonnegative defined integer $m$, mostly we can find one $w_{k}$, for which

$$
1-\frac{1}{2^{m}} \leq h\left(w_{k}\right)<1-\frac{1}{2^{m+1}} .
$$

Then, there is $m_{0} \in \mathbb{N}$, such that for any concerned Carleson window,

$$
\Omega=\left\{r e^{i \theta}: 0<1-r<l(\Omega),\left|\theta-\theta_{0}\right|<l(\Omega)\right\}
$$

and $m \in \mathbb{N}$; thus, we can almost find $m_{0}$ elements in

$$
\left\{h\left(w_{k}\right) \in \Omega: 2^{-m-1} l(\Omega)<1-\left|h\left(w_{k}\right)\right|<2^{-m} l(\Omega)\right\} .
$$

Hence, $\left(h\left(w_{k}\right)\right)_{k \in \mathbb{N}}$ is an interpolating concerned specific sequence for the analytic space $B^{s}$.

Then, we may have some certain functions $P(w) \in B^{s}$, for which

$$
P\left(h\left(w_{k}\right)\right)=\log \frac{1}{1-\left|h\left(w_{k}\right)\right|^{2}}, \quad k \in \mathbb{N} .
$$

Assume that $\phi(h(w))=\int_{0}^{h(w)} P(\xi) d \xi$. Therefore, in view of the definition of the analytic Bloch space $B^{s}$ and analytic Zygmund functions, we deduce that $h \in Z$. Hence, we infer that

$$
\begin{aligned}
\left(1-\left|w_{k}\right|^{2}\right)^{s}\left|\left(S_{\phi}^{g, n}\right)^{\prime}\left(w_{k}\right)\right| & =\left(1-\left|w_{k}\right|^{2}\right)^{s}\left|g^{(n-1)}\left(w_{k}\right)\right| \phi^{\prime}\left(h\left(w_{k}\right)\right) \\
& =\left(1-\left|w_{k}\right|^{2}\right)^{s}\left|g^{(n-1)}\left(w_{k}\right)\right| P\left(h\left(w_{k}\right)\right) \\
& =\left(1-\left|w_{k}\right|^{2}\right)^{s}\left|g^{(n-1)}\left(w_{k}\right)\right| \log \frac{1}{1-\left|h\left(w_{k}\right)\right|^{2}} \\
& \geq \varepsilon_{0}>0 .
\end{aligned}
$$

Because $\lim _{k \longrightarrow \infty}\left|h\left(w_{k}\right)\right|=1$ which yields that $\lim _{k \rightarrow \infty}\left|w_{k}\right|$ $=1$, therefore, using the above inequality, we deduce that $S_{\phi}^{g, n} \in B_{\omega, 0}^{s}$, which is a contradiction. The proof of Theorem 12 is completely finished. 
Theorem 13. Suppose that $h, g \in H(\mathbb{D})$ also assumes that $\phi$ defines a concerned entire-type function. Thus, the considered operator $S_{\phi}^{g, n}: B_{v} \longrightarrow B_{v}$ is a compact operator iff

$$
\left\|S_{\phi}^{g, n} \varphi_{b}\right\|_{B_{v}} \longrightarrow 0, \quad \text { as }|b| \longrightarrow 1
$$

Proof. Assuming that the compactness property for the operator $S_{\phi}^{g}: B_{v} \longrightarrow B_{v}$ is satisfied. Therefore, $\left\{\varphi_{b}(w): b \in \mathbb{D}\right\}$ can be viewed as a concerned bounded set in the analytic Bloch space $B_{v}$ and the specific function $\varphi_{b}-b \longrightarrow 0$, where the convergence is of uniform type on specific compact concerned sets when $|b| \longrightarrow 1$.

Hence, applying Lemma 8, we deduce that

$$
\lim _{|b| \longrightarrow 1}\left\|S_{\phi}^{g, n} \varphi_{b}\right\|_{B_{v}}=0
$$

On the other hand, assume that

$$
\lim _{|b| \longrightarrow 1}\left\|S_{\phi}^{g, n} \varphi_{b}\right\|_{B_{v}}=0, \quad \text { as }|b| \longrightarrow 1 .
$$

Let $\left(h_{n}\right)$ be a concerned bounded sequence in the analytic-type space $B_{v}$, for which $h_{n} \longrightarrow 0$ is uniformly bounded on concerned specific compact sets, when $n \longrightarrow$ $\infty$. Then, we can clear that

$$
\lim _{n \longrightarrow 0}\left\|S_{\phi}^{g, n} h_{n}\right\|_{B_{v}}=0
$$

Assume that $\varepsilon>0$ is given also fixing $0<\delta<1$, for which $|b|>\delta$; hence, we obtain

$$
\left\|S_{\phi}^{g, n} \varphi_{b}\right\|_{B_{v}}<\varepsilon
$$

which implies that for any $w_{0} \in \mathbb{D},\left|\phi\left(w_{0}\right)\right|>\delta$ and $\left\|S_{\phi}^{g, n} \varphi_{\phi\left(w_{0}\right)}\right\|_{\mathscr{B}}<\varepsilon$.

Particularly, we have

$$
\left|\phi^{\prime}\left(\varphi_{\phi\left(w_{0}\right)}\right)\right|\left|g^{(n-1)}\left(w_{0}\right)\right| v\left(\left|w_{0}\right|\right)<\varepsilon .
$$

Therefore, considering $n \in \mathbb{N}$ and $w_{0} \in \mathbb{D}$, as well as $\mid \phi$ $\left(w_{0}\right) \mid>\delta$, (70) gives

$$
\left\|S_{\phi}^{g, n} h_{n}\right\|_{B_{v}}=\sup _{w \in \mathbb{D}}\left|\phi^{\prime}\left(h_{n}\left(w_{0}\right)\right)\right|\left|g^{(n-1)}\left(w_{0}\right)\right| \nu\left(\left|w_{0}\right|\right) \leq \varepsilon \text { const. }
$$

The concerned set $K_{1}=\{z:|z| \leq \delta\}$ is a concerned compact subset of the disk $\mathbb{D}$. Also, the convergence of $\phi_{n}$ ' $\longrightarrow 0$ is uniformly bounded on specific compact concerned sets:

$$
\sup _{z \in K_{1}}\left|\phi_{n}^{\prime}(z)\right| \longrightarrow 0, \quad \text { when } n \longrightarrow \infty
$$

Hence, we can find $n_{0}$ which can be chosen to be large enough; then, $\left|\left(\phi^{\prime}\left(h_{n}\right)\right)\right|<\varepsilon$, for any $n>n_{0}$, with $z \in \mathbb{D}$, for which $|h(z)| \leq \delta$. Hence,

$$
\begin{aligned}
\left\|S_{\phi}^{g, n} h_{n}\right\|_{B_{v}} & =\left|\left(\phi^{\prime}\left(h_{n}\right)\right) \| g^{(n-1)}(z)\right| v(|z|) \\
& <\varepsilon\left|g^{(n-1)}(z)\right| v(|z|) \\
& \leq \varepsilon\|g\|_{B_{v}} \\
& <\varepsilon \text { const. }
\end{aligned}
$$

Then, where $n \geq n_{0}$,

$$
\left\|S_{\phi}^{g} f_{n}\right\|_{B_{v}}<\varepsilon \text { const. }
$$

Therefore, combining equations (71) and (74), we deduce that

$$
\left\|S_{\phi}^{g, n} h_{n}\right\|_{B_{v}}<\varepsilon \text { const., for } n \geq n_{0}
$$

Therefore, (75) gives $\left\|S_{\phi}^{g, n} h_{n}\right\|_{B_{v}} \longrightarrow 0$ as $n \longrightarrow \infty$. Hence, in view of the concerned Lemma 8, we deduce that the operator $S_{\phi}^{g, n}: B_{v} \longrightarrow B_{v}$ is actually a compact operator.

Corollary 14. Let $0<s<1$ and $h, g \in H(\mathbb{D})$ and let $\phi$ be a holomorphic-type self-mapping on $\mathbb{D}$. Thus, $S_{\phi}^{g, n}: B_{v} \longrightarrow B^{s}$ is a compact operator iff

$$
\left\|S_{\phi}^{g, n} \varphi_{b}\right\|_{B_{v}} \longrightarrow 0, \quad a s|b| \longrightarrow 1
$$

Proof. The proof of this corollary can be obtained immediately from the proof of Theorem 13 by setting $v(|w|)=$ $(1-|w|)^{s}, 0<s<1$, in the image of the operator $S_{\phi}^{g, n}$.

\section{Conclusions}

An interesting approach for defining and discussing a new class of integral superposition-type operators is provided in this concerned manuscript. This class of operators is based on both an inducing entire function and a concerned analytic function of " $n-1$ "-order derivatives, where $n \in \mathbb{N}$.

Essential results of the nonlinear $n$-generalized superposition operator defined for the first time are introduced in the present manuscript. Using the new class of operators, certain classical and recent classes of analytic-type function spaces such as Zygmund and $v$-Bloch classes are studied. Careful foundations for the concerned weighted analytictype function class theoretic results are devoted to laying and discussing properties of the operator $S_{\phi}^{g, n}$ such as the usual properties of boundedness and compactness. Both the new operator $S_{\phi}^{g, n}$ and the concerned analytic function space ultraproduct constructions are studied in detail. 
For the $n$-generalized superposition operator $S_{\phi}^{g, n}$, the next useful important remarks are stated as unsolved interesting future research.

Remark 15. Can one discuss some certain specific properties such as boundedness and compactness for the operator $S_{\phi}^{g, n}$ on some other types of analytic function spaces such as Besov- and Q-type spaces?

Remark 16. How about estimating the essential norms of the $n$-generalized superposition operator $S_{\phi}^{g, n}$ ?

In addition, there are some interesting recent studies on some types of Morrey spaces by applying some certain classes of operators (see $[19,20])$. Is it possible to apply the $n$-generalized superposition operator $S_{\phi}^{g, n}$ in lieu of the considered operators in such studies?

Remark 17. There are some recent interesting and important studies of some hypercomplex function spaces using quaternion techniques (see [21-24]). On the other hand, there is no specific study with the help of operators on such hypercomplex spaces.

\section{Data Availability}

The authors do not have any data during this concerned study.

\section{Conflicts of Interest}

The authors of this current research are clearly declaring that this study has been done without any competing interests.

\section{Acknowledgments}

The first author is deeply thankful to Taif University Researchers for supporting the study (Project number TURSP-2020/159, Taif University, Saudi Arabia).

\section{References}

[1] A. El-Sayed Ahmed and S. Omran, "Weighted superposition operators in some analytic function spaces," Journal of Computational Analysis and Applications, vol. 15, no. 6, pp. 9961005, 2013.

[2] V. Álvarez, M. A. Márquez, and D. Vukotić, "Superposition operators between the Bloch space and Bergman spaces," Arkiv för Matematik, vol. 42, no. 2, pp. 205-216, 2004.

[3] J. Appell and P. P. Zabrejko, Nonlinear Superposition Operators, Cambridge University Press, Cambridge, 2012.

[4] S. M. Buckley, J. L. Fernández, and D. Vukotić, "Superposition operators on Dirichlet type spaces," in Papers on Analysis: A Volume Dedicated to Olli Martio on the Occasion of His 60th Birthday, Rep. Univ. Jyvaskylä Dept. Math. Stat, vol. 83, pp. 41-61, University of Jyväskylä, Jyväskylä, 2001.

[5] S. M. Buckley and D. Vukotic, "Univalent interpolation in Besov spaces and superposition into Bergman spaces," Potential Analysis, vol. 29, no. 1, pp. 1-16, 2008.
[6] G. A. Cámera, "Nonlinear superposition on spaces of analytic functions," in Harmonic Analysis and Operator Theory (Caracas, 1994), Contemp. Math, vol. 189, pp. 103-116, Amer. Math. Soc., Providence, RI, 1995.

[7] G. A. Cámera and J. Gimenez, "The nonlinear superposition operator acting on Bergman spaces," Compositio Mathematica, vol. 93, pp. 23-35, 1994.

[8] S. Dominguez and D. Girela, "Sequences of zeros of analytic function spaces and weighted superposition operators," Monatshefte für Mathematik, vol. 190, no. 4, pp. 725-734, 2019.

[9] Z. J. Jiang, T. Wang, J. Liu, J. Luo, and T. Song, "Weighted superposition operators from Zygmund space to $\mu$-Bloch spaces," Journal of Computational Analysis and Applications, vol. 23, no. 3, pp. 487-495, 2017.

[10] Y. X. Liang and Z. H. Zhou, "The nonlinear superposition operators between Zygmund-type and Bloch-type spaces," Mediterranean Journal of Mathematics, vol. 16, no. 2, 2019.

[11] R. J. Malavé-Malavé and J. C. Ramos-Fernández, "Superposition operators between logarithmic Bloch spaces," Rendiconti del Circolo Matematico di Palermo Series 2, vol. 68, no. 1, pp. 105-121, 2019.

[12] R. J. Malavé-Malavé and J. C. Ramos-Fernández, “The integral weight and superposition operators between Bloch-type spaces," Bulletin of the Malaysian Mathematical Sciences Society, vol. 43, no. 4, pp. 3035-3047, 2020.

[13] C. Xiong, "Superposition operators between $Q p$ spaces and Bloch-type spaces," Complex Variables, Theory and Application: An International Journal, vol. 50, no. 12, pp. 935-938, 2006.

[14] W. Xu, "Superposition operators on Bloch-type spaces," Computational Methods and Function Theory, vol. 7, no. 2, pp. 501-507, 2007.

[15] P. Duren, Theory of $H^{p}$ Spaces, Academic Press, New York, 1973.

[16] W. Rudin, Real and Complex Analysis, McGraw-Hill, New York, 1987

[17] M. Tjani, "Compact composition operators on Besov spaces," Transactions of the American Mathematical Society, vol. 355, no. 11, pp. 4683-4698, 2003.

[18] J. B. Conway, Functions of One Complex Variable, SpringerVerlag, New York, Second edition, 1978.

[19] F. Gürbüz, "Some estimates for generalized commutators of rough fractional maximal and integral operators on generalized weighted Morrey spaces," Canadian Mathematical Bulletin, vol. 60, no. 1, pp. 131-145, 2017.

[20] F. Gürbüz, "On the behaviors of rough multilinear fractional integral and multi-sublinear fractional maximal operators both on product $L^{p}$ and weighted $L^{p}$ spaces," International Journal of Nonlinear Sciences and Numerical Simulation, vol. 21, no. 7-8, pp. 715-726, 2020.

[21] A. El-Sayed Ahmed, "Hyperholomorphic Q classes," Mathematical and Computer Modelling, vol. 55, no. 3-4, pp. 14281435, 2012.

[22] A. El-Sayed Ahmed, "Characterizations for general Besov-type space in Clifford analysis," Advances in Applied Clifford Algebras, vol. 24, no. 4, pp. 1011-1025, 2014.

[23] A. El-Sayed Ahmed and S. Omran, "Weighted classes of quaternion-valued functions," Banach Journal of Mathematical Analysis, vol. 6, no. 2, pp. 180-191, 2012.

[24] A. El-Sayed Ahmed and S. Omran, "Extreme points and some quaternion valued functions in the unit ball of $\mathbb{R}^{3}$," Advances in Applied Clifford Algebras, vol. 28, no. 1, p. 31, 2018. 